\title{
Dopant and Interfacial Analysis of Epitaxial CdTe Using Atom Probe Tomography
}

\author{
George L. Burton ${ }^{1}$, David R. Diercks ${ }^{1}$ and Brian P. Gorman ${ }^{1}$ \\ ${ }^{1 .}$ Metallurgical and Materials Engineering, Colorado School of Mines, Golden, CO.
}

Cadmium Telluride (CdTe) is a II-VI semiconductor with a direct band gap of $1.45 \mathrm{eV}$ at room temperature. It has a high absorption coefficient and can be produced easily from raw materials, making it an ideal material for photovoltaic (PV) devices. Thin film polycrystalline CdTe PV devices have shown exceptional promise, reaching efficiencies of 20\% [1], however much research must be accomplished to approach the theoretical efficiency of $\sim 30 \%$. The study of polycrystalline CdTe is hindered primarily by grain boundaries (GBs), which give rise to high recombination rates, current leakage, device shunting, and enhanced migration of dopants [2].

To overcome the complexity and problems associated with GBs, CdTe can be grown in a single crystal form with meticulous control over chemical purity and dopant concentration through molecular beam epitaxy (MBE). Identifying the concentration, uniformity, and location of impurity species, whether added intentionally (i.e. dopants) or unintentionally (i.e. unwanted contaminants), can help to optimize the MBE growth parameters for high efficiency devices. With its unparalleled three-dimensional atomic spatial resolution, atom probe tomography (APT) is particularly suited for determining these impurity properties.

Laser-pulsed APT in conjunction with correlative transmission electron microscopy (TEM) was conducted on a number of epitaxial CdTe thin films. TEM images of specimens taken prior to and following APT were used to produce an accurate analysis volume for APT reconstructions [3]. A variety of dopant species were analyzed, including arsenic as shown in Figure 1. The APT reconstruction shows clustering of As, with local concentrations sometimes exceeding 40 at. \%, throughout the sample. Additionally, the concentration profile suggests that As is unexpectedly substituting for Cd rather than Te, which implies n-type doping rather than p-type. In Figure 2, a cadmium magnesium telluride (CdMgTe) heterojunction layer was analyzed. In addition to determining the uniformity and concentration of magnesium within the layer, APT analysis revealed a small amount of oxygen contamination. Interfacial impurities such as these can have a particularly detrimental effect on defect densities and carrier lifetimes. The relationship of the growth conditions to the structural and compositional TEM and APT findings and their impacts on material performance will be discussed.

\section{References:}

[1] Martin A. Green et al, Prog. Photovolt.: Res. Appl., 22 (2014), p. 701.

[2] S. Girish Kumar and K.S.R. Koteswara Rao, Energy Environ. Sci. 7 (2014), p. 45.

[3] B. P. Gorman et al., Microscopy Today, 16 (2008), p. 42.

[4] The authors acknowledge funding from the Department of Energy's SunShot Foundational Program to Advance Cell Efficiency (F-PACE II). 

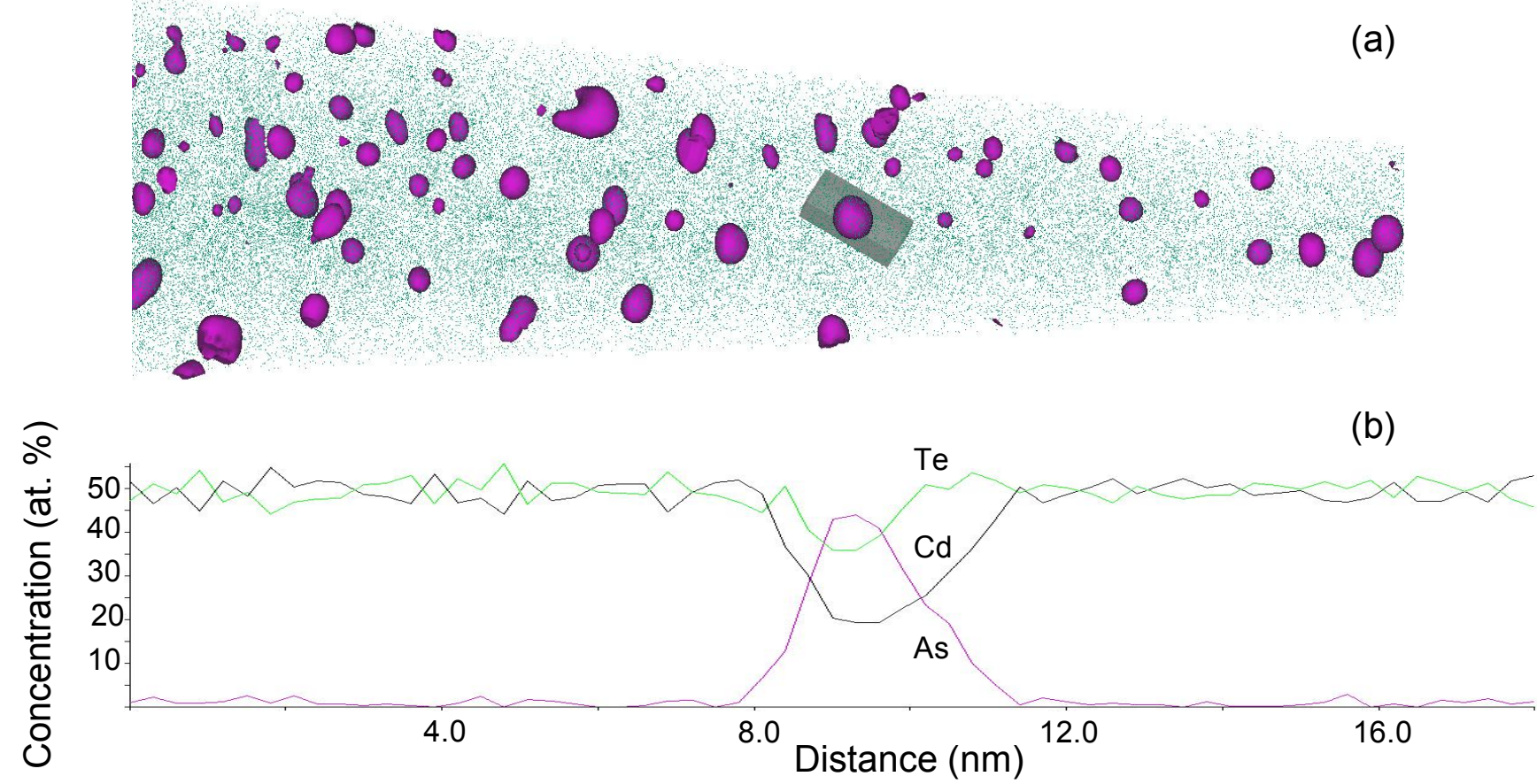

Figure 1. (a) APT reconstruction of As-doped CdTe, using 15 at. \% As isoconcentration surfaces (b) concentration profile through one As cluster whose region is defined by the grey box in the reconstruction in part (a).
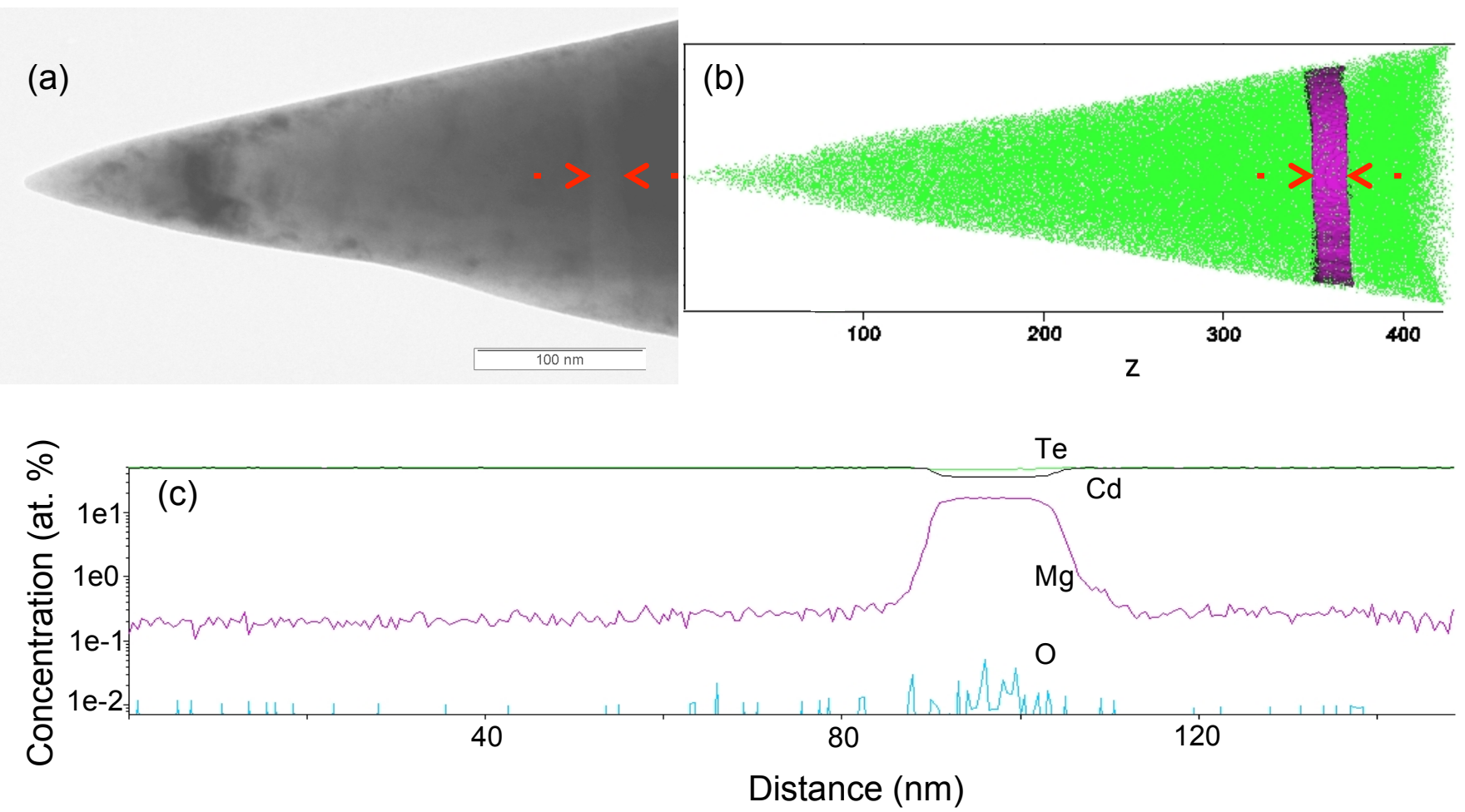

Figure 2. (a) Transmission electron micrograph of epitaxially grown CdTe with a $\sim 21 \mathrm{~nm} \mathrm{CdMgTe}$ heterojunction. (b) APT reconstruction of same sample using Mg isoconcentration surface. The green points are $\mathrm{Te}(\mathrm{c})$ Concentration of $\mathrm{Cd}, \mathrm{Te}, \mathrm{Mg}$, and $\mathrm{O}$ across the $\mathrm{CdMgTe}$ heterojunction layer. 